\title{
Terminal Sliding Mode Control for Cyber Physical System Based on Filtering Backstepping
}

\author{
Fei $\mathrm{Li}^{1} \quad$ Jian-Bo Hu${ }^{1} \quad$ Lei Zheng ${ }^{1} \quad$ Jian-Hao Wang ${ }^{2}$ \\ ${ }^{1}$ Equipment Management and Safety Engineering College, Air Force Engineering University, Xi'an 710051, China \\ ${ }^{2}$ Unit 93286, People's Liberation Army, Shenyang 110141, China
}

\begin{abstract}
Based on a nonlinear flight dynamic model with aerodynamic coefficients and external disturbance uncertainties, which is a typical cyber physical system, a filtering backstepping terminal sliding mode control method is proposed for a robust controller. The tracking differentiator can provide the capability of solving the problem of "complexity explosion" in backstepping controllers to simplify the backstepping implementation. Nonlinear disturbance observers are used to observe the uncertainties of the nonlinear flight dynamic system. The terminal sliding mode controller is designed to improve its convergence rate and the tracking accuracy. Finally, nonlinear 6-degree-of-freedom simulation results for an F-16 aircraft model elaborate the effectiveness of the proposed control system.
\end{abstract}

Keywords: Cyber-physical system (CPS), disturbance uncertainties, filtering backstepping, nonlinear disturbance observer (NDO), terminal sliding mode control.

\section{Introduction}

In recent years, cyber-physical systems, which consist of physical and computational components, have drawn considerable attention ${ }^{[1]}$. The fact that computation, communication and control (3C) are now highly integrated in the concept of cyber-physical system (CPS). A typical CPS has the capabilities of sensing, controlling and communication, which can reflect the interaction and coordination between the physical and computational elements. Therefore, the design of control strategy in CPS is of crucial importance for stabilization and efficiency of system dynamics. CPS can be found in aerospace, automotive, transportation, infrastructure management and environmental monitoring ${ }^{[2,3]}$. An important application of CPS is the flight control system ${ }^{[4]}$. And the efficacy of the control law will decide whether the CPS can bring its predominance into play or not ${ }^{[5]}$.

Due to the unmodeled dynamics and external disturbances, the flight control system is a typical uncertain nonlinear system. Recently, adaptive backstepping design method has become an important method for the research of dealing with uncertainties ${ }^{[6-8]}$. A drawback of the conventional backstepping method may cause the problem of complexity resulting from the repeated differentiations ${ }^{[9]}$. Dynamic surface control (DSC) technique could solve the complexity problem in backstepping design method by introducing a first-order filter of the synthetic input at each step of the traditional backstepping design procedure ${ }^{[10]}$. Sliding mode control (SMC) has better robustness against

Regular Paper

Manuscript received November 2, 2013; accepted November 25, 2014 This work was supported by the State Key Laboratory of Industrial Control Technology Zhejiang University China (No. ICT1447) and Shanghai Leading Academic Discipline Project (No. J50103).

Recommended by Associate Editor Yuan-Qing Xia

(C) Institute of Automation, Chinese Academy of Science and Springer-Verlag Berlin Heidelberg 2015 the parameter uncertainties ${ }^{[11-13]}$. In $[11,12], \mathrm{SMC}$ was proposed for the full envelop of the aircraft model with parameter uncertainties. The combination of SMC and adaptive backstepping control benefits from both approaches. The role of SMC is to achieve more robustness to disturbances and uncertainties ${ }^{[14]}$. However, unwanted chattering in the conventional sliding surface makes adaptive backstepping SMC unsuitable for high accuracy requirement. There are many methods which can be employed to reduce chattering such as integral sliding surface ${ }^{[15]}$, highorder $\mathrm{SMC}^{[16]}$ and dynamic $\mathrm{SMC}^{[17]}$. High-order sliding mode controllers can also be used to improve the system responses.

In this paper, the flight control CPS with aerodynamic coefficients and external disturbances is studied by adaptive backstepping SMC. The tracking differentiator is used to reduce the complexity of the computation. Nonlinear disturbance observer (NDO) is used to observe the uncertainties to compensate the control inputs ${ }^{[18,19]}$. The highorder sliding mode control law is deigned to eliminate the chattering and improve the system response time. Simulation examples are given to illustrate the effectiveness of the proposed methods.

Nomenclature.

$F$ : Aerodynamic forces about the body-fixed frame

$L, M, N$ : Aerodynamic rolling, pitching, yawing moments

I: Moment of inertia

$p, q, r$ : Roll, pitch, yaw rates about the body-fixed frame $\bar{q}$ : Dynamic pressure

$m$ : Aircraft mass

$T$ : Thrust

$S$ : Reference wing area

$C_{*}$ : Non-dimensional aerodynamic coefficient 
$V$ : Velocity

$\alpha, \beta$ : Angle of attack, sideslip angle

$\delta_{e}, \delta_{a}, \delta_{r}$ : Elevator, aileron, rudder angles

$\phi, \theta, \psi:$ Roll, pith, yaw angles

\section{Problem statement}

The body-fixed nonlinear equations of motion for an F-16 aircraft with external uncertainties and aerodynamic coefficients can be written as ${ }^{[20]}$

$$
\left\{\begin{array}{l}
\dot{x}_{1}=f_{1}\left(x_{1}, x_{3}\right)+g_{1}\left(x_{1}, x_{3}\right) x_{2}+M_{1} \\
\dot{x}_{2}=f_{2}\left(x_{1}, x_{2}\right)+g_{2}\left(x_{1}\right) u+M_{2} \\
\dot{x}_{3}=f_{3}\left(x_{1}, x_{2}\right) \\
M_{1}=\Delta f_{1}\left(x_{1}\right)+\Delta g_{1}\left(x_{1}\right) x_{2}+\left[h\left(x_{1}\right)+\Delta h\left(x_{1}\right)\right] u \\
M_{2}=\Delta f_{2}\left(x_{1}, x_{2}\right)+\Delta g_{2}\left(x_{1}\right) u+d(t)
\end{array}\right.
$$

$x_{1}=[\alpha, \beta, \phi]^{\mathrm{T}}, x_{2}=[p, q, r]^{\mathrm{T}}, x_{3}=[\theta, \psi]^{\mathrm{T}}, u=\left[\delta_{e}, \delta_{a}, \delta_{r}\right]^{\mathrm{T}}$.

And the expressions of $f_{1}, g_{1}, f_{2}, g_{2}, f_{3}, h$ are the same as given in [20].

$M_{i}$ represents the compound uncertainties in the model. $\Delta f, \Delta g$ and $\Delta h$ represent the aerodynamic coefficients, $d(t)$ is the external uncertainty. $h$ represents the aerodynamic force component caused by the control surface deflection. It is seen as one part of the model uncertainties $M_{1}$. The task of the controller to be designed is to track the commands of $\alpha, \beta$ and $\phi$. Assumption 1 is used in the design and analysis process.

Assumption 1. The desired trajectory $y_{c}=\left[\alpha_{c}, \beta_{c}\right.$, $\left.\phi_{c}\right]^{\mathrm{T}}$ is bounded, namely

$$
\left\|\left[y_{c}, \dot{y}_{c}, \ddot{y}_{c}\right]\right\| \leq c_{0}
$$

where $c_{0} \in \mathbf{R}$ is a known positive constant and $\|\cdot\|$ denotes the 2-norm of a vector or a matrix.

Lemmas 1 and 2 are used in the design and analysis process.

Lemma 1. There exist positive constants $\alpha_{m}, \beta_{m}$ and $\theta_{m} \in \mathbf{R}$ such that the magnitudes and derivatives of $f_{1}, f_{2}, g_{1}$ and $g_{2}$ are bounded for all $\alpha, \beta$ and $\theta \in \mathbf{R}$ satisfying $|\alpha| \leq \alpha_{m}$ and $|\beta| \leq \beta_{m}$. Furthermore, there exist $g_{i 0}$ and $g_{i 1}$ such that $0<g_{i 0} \leq\left\|g_{i}\right\| \leq g_{i 1}, i=1,2$.

Lemma 2. There exist two arbitrary vectors $x, y \in \mathbf{R}^{n}$ satisfying

$$
x^{\mathrm{T}} y \leq \frac{\varepsilon^{p}}{p}\|x\|^{p}+\frac{1}{q \varepsilon^{q}}\|y\|^{q}
$$

$\varepsilon>0, p>1, q>1$ and $(p-1)(q-1)=1$. When $p=q=2$ and $\varepsilon^{2}=2$, there exist $x, y \in \mathbf{R}^{n}$ such that $x^{\mathrm{T}} y \leq\|x\|^{2}+$ $\|y\|^{2}$.

\section{Controller design and stability anal- ysis}

\subsection{Tracking differential filter}

The derivative of the virtual control signal obtained through the filter can effectively reduce the amount of calculation in the backstepping control. The classical differential filter has amplification effect on noises. Nonlinear tracking differentiator (NTD) can overcome the drawback when it is used to simplify the calculation. Its expression is written as $^{[21]}$

$$
\left\{\begin{array}{l}
\dot{y}_{1}=y_{2} \\
\dot{y}_{2}=\gamma \operatorname{sat}\left(y_{1}-v_{0}(t)+\frac{y_{2}\left|y_{2}\right|}{2 \gamma}, \delta\right)
\end{array}\right.
$$

where $v_{0}(t)$ is the input signal, $y_{1}$ is the tracking signal, $y_{2}$ is the approximate differential signal of $v_{0}(t), \operatorname{sat}(x, \delta)$ is the saturation function, i.e.,

$$
\operatorname{sat}(x, \delta)=\left\{\begin{array}{c}
-1, x<-\delta \\
\frac{x}{\delta},|x| \leq \delta \\
1, x>\delta
\end{array}\right.
$$

$\gamma>0$, and the value of $\gamma$ determines the tracking speed of NTD. From (2), the following equality is satisfied when $v_{0}(t)$ is bounded and $\gamma$ is sufficiently large

$$
\lim _{\gamma \rightarrow \infty} \int_{0}^{T}\left|y_{1}(t)-v_{0}(t)\right| \mathrm{d} t=0
$$

with $T>0$. We can know that NTD does not affect the convergence of the tracking error of the control system ${ }^{[22]}$. And $y_{2}$ is obtained by integrating the differential signal, which can effectively avoid the influence of noise.

\subsection{Controller design}

Define the tracking error of the states in the control system

$$
\left\{\begin{array}{l}
e_{1}=x_{1}-x_{1 c} \\
e_{2}=x_{2}-x_{2 c}
\end{array}\right.
$$

where $x_{1 c}=y_{c}, x_{2 c}$ is the expected virtual input of the inner loop. The derivative of $e_{1}$ is

$$
\dot{e}_{1}=\dot{x}_{1}-\dot{e}_{1 c}=f_{1}+g_{1} x_{2}+M_{1}-\dot{y}_{c} .
$$

NDO is adopted to estimate uncertainty $M_{1}$, then

$$
\left\{\begin{array}{l}
\hat{M}_{1}=z_{1}+p_{1} \\
\dot{z}_{1}=-L_{1} z_{1}-p_{1}\left(L_{1}+f_{1}+g_{1} x_{2}\right)
\end{array}\right.
$$

where $L_{1}=\operatorname{diag}\left\{\begin{array}{lll}L_{11} & L_{12} & L_{13}\end{array}\right\}$ is a positive constant, $p_{1}$ is a nonlinear function to be designed, and $L_{1}=\partial p_{1}(x) / \partial x$. The virtual control $\bar{x}_{2 c}$ to drive $e_{1} \rightarrow 0$ based on NDO can be determined as

$$
\bar{x}_{2 c}=-g_{1}^{-1}\left(k_{1} e_{1}+f_{1}+\hat{M}_{1}-\dot{y}_{c}\right)
$$

where $k_{1}=\operatorname{diag}\left\{\begin{array}{llll}k_{11} & k_{12} & k_{13}\end{array}\right\}$ is a positive constant. In order to obtain the filtering virtual control $x_{2 c}$, we pass $\bar{x}_{2 c}$ through an NTD with $\gamma>0$. The derivative of $e_{2}$ is

$$
\dot{e}_{2}=\dot{x}_{2}-\dot{x}_{2 c}=f_{2}+g_{2} u+M_{2}-\dot{x}_{2 c} .
$$

The NDO of the inner loop is given by

$$
\left\{\begin{array}{l}
\hat{M}_{2}=z_{2}+p_{2} \\
\dot{z}_{2}=-L_{2} z_{2}-L_{2}\left(p_{2}+f_{2}+g_{2} u\right) .
\end{array}\right.
$$


Define a non-singular terminal sliding mode surface as follows:

$$
S=\left[S_{1}, S_{2}, S_{3}\right]^{\mathrm{T}}=e_{2}+a \dot{e}_{2}^{\frac{\rho_{1}}{\rho_{2}}}
$$

where $a=\operatorname{diag}\left\{\begin{array}{lll}a_{1} & a_{2} & a_{3}\end{array}\right\}, a_{i}>0, \rho_{1}$ and $\rho_{2}$ are positive odd constants to be designed and $1<\frac{\rho_{1}}{\rho_{2}}<2$, $\dot{e}_{2}^{\frac{\rho_{1}}{\rho_{2}}}=\left[\dot{e}_{21}^{\frac{\rho_{1}}{\rho_{2}}}, \dot{e}_{22}^{\frac{\rho_{1}}{\rho_{2}}}, \dot{e}_{23}^{\frac{\rho_{1}}{\rho_{2}}}\right]^{\mathrm{T}}$. Let $S(t)=0, t \geq t_{r}$, then

$$
\lim _{t \rightarrow t_{s}} e_{2}=0, \quad \lim _{t \rightarrow t_{s}} \dot{e}_{2}=0
$$

where

$$
t_{s}=t_{r}+\frac{\rho_{1}}{\rho_{1}-\rho_{2}} \max _{1 \leq i \leq 3}\left(a_{i}^{\frac{\rho_{1}}{\rho_{2}}}\left\|e_{2 i}\left(t_{r}\right)\right\|^{\frac{\rho_{1}-\rho_{2}}{\rho_{2}}}\right) .
$$

Therefore, the system will remain in the second-order sliding mode when $t \geq t_{s}$. The values of $\rho_{1}, \rho_{2}, a$ determine the speed of convergence of the control system. In this paper, a high-order terminal sliding mode control law is proposed as

$$
\left\{\begin{array}{l}
u=u_{1}+u_{2} \\
u_{1}=-g_{2}^{-1}\left(f_{2}+\hat{M}_{2}-\dot{x}_{2 c}\right) \\
u_{2 i}=-\left(g_{2}^{-1}\right)_{i} \int_{0}^{t}\left[\frac{\rho_{2}}{a_{i} \rho_{1}} \dot{e}_{2 i}^{2-\frac{\rho_{1}}{\rho_{2}}}+k_{2 i} S_{i}+k_{3 i} \operatorname{sgn}\left(S_{i}\right)\right] \mathrm{d} \tau \\
u_{2}=\left[u_{21}, u_{22}, u_{23}\right]^{\mathrm{T}}
\end{array}\right.
$$

where

$$
\begin{array}{ll}
k_{2}=\operatorname{diag}\left\{k_{21}, k_{22}, k_{23}\right\}, & k_{2 j}>0, \quad j=1,2,3 \\
k_{3}=\operatorname{diag}\left\{k_{31}, k_{32}, k_{33}\right\}, & k_{3 j}>0, \quad j=1,2,3 .
\end{array}
$$

\subsection{Stability analysis}

It can be assumed that the disturbances are slowly time-varying, so we can consider that $\dot{M} \approx 0$ and $\dot{e}_{\mathrm{NDO}}=-L(x) e_{\mathrm{NDO}}^{[19]}$.

Consider the following Lyapunov function candidate:

$$
V_{1}=\frac{1}{2} e_{1}^{\mathrm{T}} e_{1}+\frac{1}{2} e_{\mathrm{NDO} 1}^{\mathrm{T}} e_{\mathrm{NDO} 1}
$$

The derivative of $V_{1}$ is

$$
\begin{aligned}
\dot{V}_{1}= & e_{1}^{\mathrm{T}} \dot{e}_{1}+e_{\mathrm{NDO} 1}^{\mathrm{T}} \dot{e}_{\mathrm{NDO} 1}= \\
& e_{1}^{\mathrm{T}}\left(-k_{1} e_{1}+g_{1} e_{2}+e_{\mathrm{NDO} 1}\right)-e_{\mathrm{NDO} 1}^{\mathrm{T}} L_{1} e_{\mathrm{NDO} 1}
\end{aligned}
$$

From Lemma 2,

$$
\begin{gathered}
\dot{V}_{1} \leq-\lambda_{\min }\left(k_{1}-2 I\right)\left\|e_{1}\right\|^{2}+e_{1}^{\mathrm{T}} g_{1} e_{2}- \\
\lambda_{\min }\left(L_{1}-\frac{1}{4} I\right)\left\|e_{\mathrm{NDO} 1}\right\|^{2} .
\end{gathered}
$$

Let $k_{1}>I$ and $L_{1}>\frac{1}{4} I$. Then,

$$
\dot{V}_{1} \leq 0 \text {. }
$$

Therefore, we can know that $e_{1}$ and $e_{\mathrm{NDO}}$ are uniformly ultimately bounded if $\lim _{t \rightarrow \infty} e_{2}(\mathrm{t})=0$. Consider the following Lyapunov function candidate

$$
V_{2}=\frac{1}{2} S^{\mathrm{T}} S+\frac{1}{2} e_{\mathrm{NDO} 2}^{\mathrm{T}} e_{\mathrm{NDO} 2} .
$$

The derivative of $V_{2}$ is

$$
\dot{V}_{2}=S^{\mathrm{T}} \dot{S}+e_{\mathrm{NDO} 2}^{\mathrm{T}} \dot{e}_{\mathrm{NDO} 2} .
$$

From (11),

$$
\dot{S}_{i}=a_{i} \frac{\rho_{1}}{\rho_{2}} \dot{e}_{2 i}^{\frac{\rho_{1}}{\rho_{2}}-1}\left(\ddot{e}_{2 i}+\frac{\rho_{2}}{a_{i} \rho_{1}} \dot{e}_{2 i}^{2-\frac{\rho_{1}}{\rho_{2}}}\right) .
$$

So,

$$
\dot{V}_{2}=\sum_{i=1}^{3}\left[S_{i} a_{i} \frac{\rho_{1}}{\rho_{2}} \dot{e}_{2 i}^{\frac{\rho_{1}}{\rho_{2}}-1}\left(\ddot{e}_{2 i}+\frac{\rho_{2}}{a_{i} \rho_{1}} \dot{e}_{2 i}^{2-\frac{\rho_{1}}{\rho_{2}}}\right)-L_{2 i} e_{\mathrm{NDO} 2}^{2}\right] .
$$

Substituting (14) into (9) yields

$$
\dot{e}_{2}=g_{2} u_{2} .
$$

The derivative of $\dot{e}_{2}$ is

$$
\ddot{e}_{2 i}=-\frac{\rho_{2}}{a_{i} \rho_{1}} \dot{e}_{2}^{2-\frac{\rho_{1}}{\rho_{2}}}-k_{2 i} S_{i}-k_{3 i} \operatorname{sgn}\left(S_{i}\right) .
$$

Substituting (24) into (20) yields

$\dot{V}_{2}=$

$\sum_{i=1}^{3}\left[S_{i} a_{i} \frac{\rho_{1}}{\rho_{2}} \dot{e}_{2 i}^{\frac{\rho_{1}}{\rho_{2}}-1}\left[-k_{2 i} S_{i}-k_{3 i} \operatorname{sgn}\left(S_{i}\right)\right]-L_{2 i} e_{\mathrm{NDO} 2}^{2}\right]=$

$-\sum_{i=1}^{3}\left[a_{i} k_{2 i} \frac{\rho_{1}}{\rho_{2}} \dot{e}_{2 i}^{\frac{\rho_{1}}{\rho_{2}}-1} S_{i}^{2}+a_{i} k_{3 i} \frac{\rho_{1}}{\rho_{2}} \dot{e}_{2 i}^{\frac{\rho_{1}}{\rho_{2}}-1}\left|S_{i}\right|+L_{2 i} e_{\mathrm{NDO} 2}^{2}\right]$.

Let $1<\frac{\rho_{1}}{\rho_{2}}<2, k_{2}>0, k_{3}>0, L_{2}>0$ and $a>0$. Then,

$$
\dot{V}_{2} \leq 0 \text {. }
$$

Accordingly, we can know that the tracking errors of the control system designed in this paper are locally uniformly ultimately bounded when $k_{1}>I, k_{2}>0, k_{3}>0, L_{1}>\frac{1}{4} I$, $L_{2}>0$ and $1<\frac{\rho_{1}}{\rho_{2}}<2$.

\section{Simulation results}

The proposed controller is tested by a numerical simulation on the attitude maneuver flight control system of an F-16 aircraft. The following command values of $\alpha_{d}, \beta_{d}$, and $\phi_{d}$ are applied to the aircraft in a steady-state level flight of $V=200 \mathrm{~m} / \mathrm{s}, h=4000 \mathrm{~m}, F_{T}=60 \mathrm{kN}$ :

$$
\begin{gathered}
\alpha_{d}=2.659, \beta_{d}=0, \phi_{d}=0,0 \leq t<1 \\
\alpha_{d}=10, \beta_{d}=0, \phi_{d}=50,1 \leq t<10 \\
\alpha_{d}=-2, \beta_{d}=0, \phi_{d}=0,10 \leq t<20 .
\end{gathered}
$$

$y_{c}$ is obtained from $y_{d}$ by the following command filter to satisfy Assumption 1:

$$
\frac{\left[y_{c}\right]_{i}}{\left[y_{d}\right]_{i}}=\frac{\omega_{n}^{2}}{s^{2}+2 s \xi_{n} \omega_{n}+\omega_{n}^{2}}, \omega_{n}=4, \xi_{n}=0.8, i=1,2,3
$$

where $s$ is Laplace operator. The controller design parameters are chosen as $k_{1}=10 I, k_{2}=5 I, \gamma=10 I, a=5 I$, 
$\tau_{2}=0.05, \delta(0)=10$, where $I$ represents the $3 \times 3$ identity matrix. NDO gains are chosen as

$$
\begin{aligned}
& L_{1}=\operatorname{diag}\left\{2\left(1+\alpha^{2}\right), 2\left(1+\beta^{2}\right), 2\left(1+\phi^{2}\right)\right\} \\
& L_{2}=\operatorname{diag}\left\{5\left(1+p^{2}\right), 5\left(1+q^{2}\right), 5\left(1+r^{2}\right)\right\} .
\end{aligned}
$$

We assume that the uncertainties of the aerodynamic coefficients and external moment disturbance are timevarying. The time-varying uncertainties are given as

$$
C_{j}=[1+0.5 \sin (0.5 \pi t)] C_{j}^{r}, j=x, y, z, l, m, n
$$

$$
d(t)=\left[\begin{array}{lll}
0.5 & 1.2 & 1.5
\end{array}\right] \times 10^{4} \sin (t)
$$

where $C_{x}^{r}, C_{y}^{r}, C_{z}^{r}, C_{l}^{r}, C_{m}^{r}$ and $C_{n}^{r}$ are the standard aerodynamic coefficients.

Figs. 1-3 present the simulation results when uncertainties do not exist. 0 represents the command signal. 1 represents the simulation result of the controller proposed in this paper. From Figs. 1-3, it is clear that the control law proposed in this paper can track the reference command signal effectively and make the tracking error converge.

To demonstrate the tracking performance of the proposed control law, the backstepping controller in [18] is applied contrastively. Figs. 4-9 present the simulation results when the uncertainties exist. 1 represents the simulation result of filtering backstepping terminal sliding mode controller proposed in this paper. 2 represents the simulation result of the backstepping controller proposed in [18]. The tracking error convergence curves of $e_{1}=\left[e_{1 \alpha}, e_{1 \beta}, e_{1 \phi}\right]$ are used to reflect the tracking control performance of the two controllers. The simulation results reveal that, under the condition of aerodynamic parameter perturbation and external disturbance uncertainties, the proposed control scheme can track the reference command signal more quickly and more accurately. Figs. 7-9 show that the nonsingular highorder terminal sliding mode control method can effectively weaken the chattering.

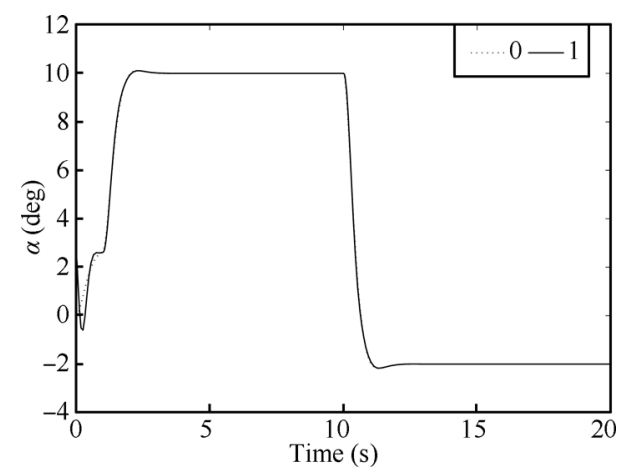

Fig. 1 Time response of $\alpha$ without uncertainties

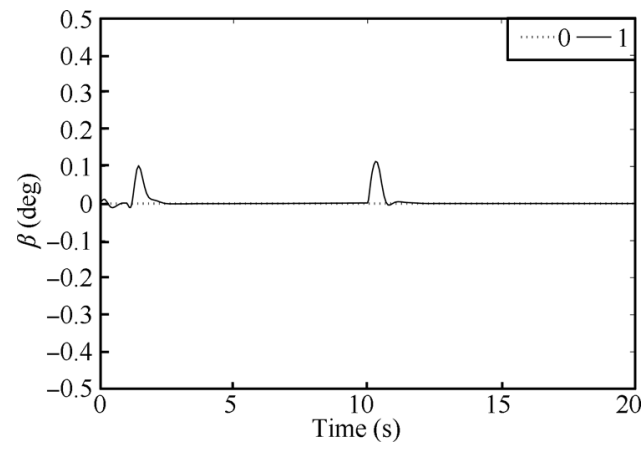

Fig. 2 Time response of $\beta$ without uncertainties

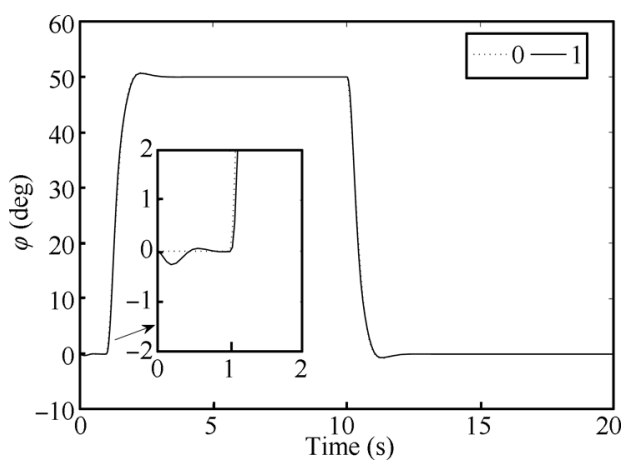

Fig. 3 Time response of $\phi$ without uncertainties

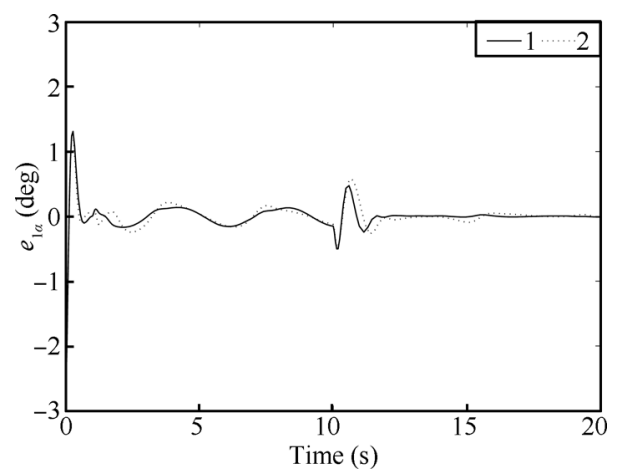

Fig. 4 Time response of $e_{1 \alpha}$ with uncertainties

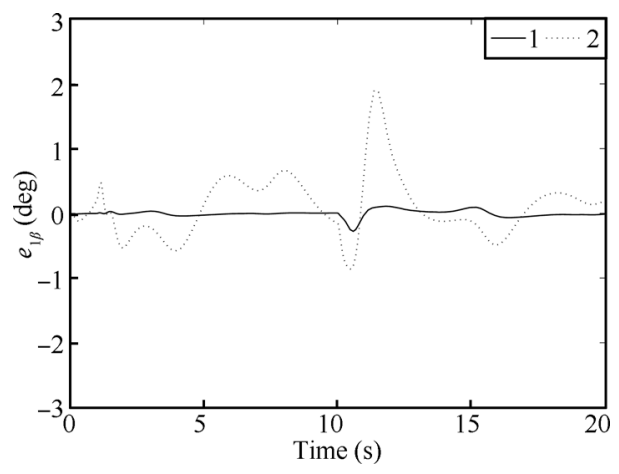

Fig. 5 Time response of $e_{1 \beta}$ with uncertainties 


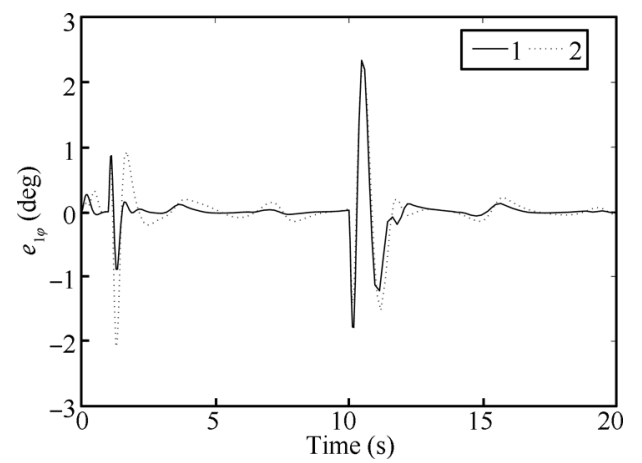

Fig. 6 Time response of $e_{1 \phi}$ with uncertainties

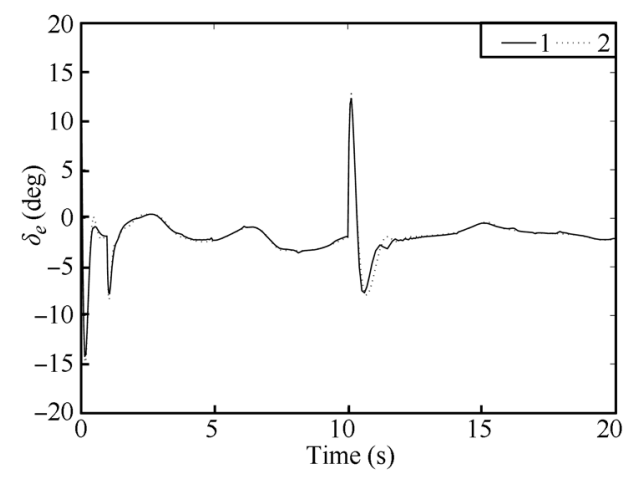

Fig. 7 Time response of $\delta_{e}$ with uncertainties

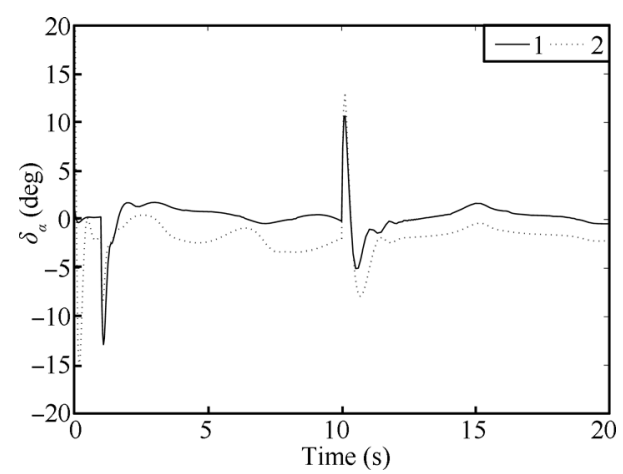

Fig. 8 Time response of $\delta_{a}$ with uncertainties

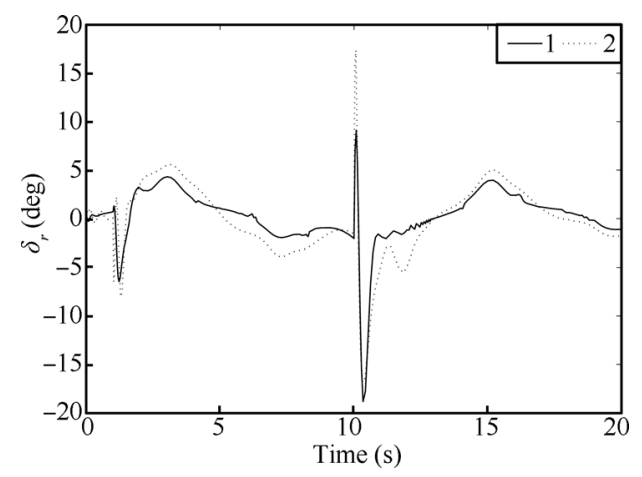

Fig. 9 Time response of $\delta_{r}$ with uncertainties

\section{Conclusions}

A filtering backstepping terminal sliding mode controller is designed in this paper to compensate for the effect of system uncertainties in CPS with time-varying parameter perturbations and external disturbances. NTD is used to obtain the derivation of the virtual control signal, simplifying the design of the controller and reducing the amount of computation. Nonlinear disturbance observers can effectively observe the uncertainties of CPS. Nonsingular terminal sliding mode controller can increase system robustness and tracking accuracy, and can effectively weaken the control signal chattering. Simulation results show that the proposed control system can quickly and accurately track the reference command signal. The method proposed in this paper may be also used in the design in other CPS controllers with aerodynamic coefficients and external disturbance uncertainties.

\section{References}

[1] H. S. Li, L. F. Lai, H. V. Poor. Multicast routing for decentralized control of cyber physical systems with an application in smart grid. IEEE Journal on Selected Areas in Communications, vol. 30, no. 6, pp. 1097-1107, 2012.

[2] J. R. Wen, M. Q. Wu, J. F. Su. Cyber-physical system. Acta Automatica Sinica, vol. 38, no. 4, pp. 507-517, 2012.

[3] R. Poovendran. Cyber-physical systems: Close encounters between two parallel worlds. Proceedings of the IEEE vol. 98, no. 8, pp. 1363-1366, 2010.

[4] M. F. Yang, L. Wang, B. Gu, L. Zhao. The application of CPS to spacecraft control system. Aerospace Control and Application, vol. 38, no. 5, pp. 8-13, 2012.

[5] H. S. Li, R. C. Qiu, Z. Q. Wu. Routing in cyber physical systems with application for voltage control in microgrids: A hybrid system approach. In Proceedings of the 32nd International Conference on Distributed Computing Systems Workshops, IEEE, Washington, USA, pp. 254-259, 2012.

[6] R. Mei, Q. X. Wu, C. S. Jiang. Robust adaptive backstepping control for a class of uncertain nonlinear systems based on disturbance observers. Science China: Information Sciences, vol. 53, no. 6, pp. 1201-1215, 2010.

[7] C. Byunghun, K. H. Jin, K. Youdan. Robust control allocation with adaptive backstepping flight control. Journal of Aerospace Engineering, vol. 228, no. 7, pp. 1033-1046, 2014.

[8] J. Chen, S. L. Zhou, Z. Q. Song. Hypersonic aircraft dynamic surface adaptive backstepping control system design based on uncertainty. Journal of Astronautics, vol. 31 , no. 11, pp. 2550-2556, 2010.

[9] S. J. Yoo. Adaptive tracking and obstacle avoidance for a class mobile robots in the presence of unknown skidding and slipping. IET Control Theory \& Applications, vol. 5, no. 14, pp. 1597-1608, 2011.

[10] J. Y. Sung, J. B. Park, Y. H. Choi. Adaptive dynamic surface control of flexible-joint robots using self-recurrent wavelet neural networks. IEEE Transactions on Systems, Man, and Cybernetics, Part B: Cybernetics, vol. 36, no. 6 , pp. 1342-1355, 2006.

[11] E. Abdlhamitbilal, E. M. Jafarov. Robust sliding mode speed hold control system design for full nonlinear aircraft 
model with parameter uncertainties: A step beyond. In Proceedings of the 12th IEEE International Workshop on Variable Structure Systems, IEEE, Mumbai, India, pp.7-15, 2012.

[12] I. C. Karagöz, E. M. Jafarov. Sliding mode robust tracking control for complete flight envelope of an UCAV with parameter uncertainties. In Proceedings of the 15th International Conference on Automatic Control, Modelling and Simulation, Brasov, Romania, pp. 253-258, 2013.

[13] J. Geng, X. D. Liu, L. Wang. Dynamic sliding mode control of a hypersonic flight vehicle. Acta Armamentarii, vol. 33, no. 3, pp. 307-312, 2012.

[14] K. Jamoussi, M. Ouali, L. Chrifi-Alaoui, H. Benderradji, A. El Hajjaji. Robust sliding mode control using adaptive switching gain for induction motors. International Journal of Automation and Computing, vol. 10, no. 4, pp.303-311, 2013.

[15] Z. Xi, T. Hesketh. Discretised integral sliding mode control for systems with uncertainty. IET Control Theory \& Applications, vol. 4, no. 10, pp. 2160-2167, 2010.

[16] J. F. Zheng, Y. Feng, X. M. Zheng, X. Q. Yang. Adaptive backstepping-based terminal-sliding-mode control for uncertain nonlinear systems. Control Theory \& Applications, vol. 26 , no. 4, pp. 410-414, 2009.

[17] L. Li, J. Xie, J. Z. Huang. Modeling and dynamic surface adaptive sliding mode control of erecting mechanism. Systems Engineering and Electronics, vol. 36, no. 2, pp. 337$342,2014$.

[18] L. P. Liu, Z. M. Fu, X. N. Song. Sliding mode control with disturbance observer for a class of nonlinear systems. International Journal of Automation and Computing, vol.9, no. 5, pp. 487-491, 2012.

[19] J. Yang, S. H. Li, C. Y. Sun, L. Guo. Nonlinear disturbance observer based robust flight control for airbreathing hypersonic vehicles. IEEE Transactions on Aerospace and Electronic Systems, vol. 49, no. 2, pp. 1263-1275, 2013.

[20] T. Y. Lee, Y. D. Kim. Nonlinear adaptive flight control using backstepping and neural networks controller. Journal of Guidance, Control, and Dynamics, vol. 24, no. 4, pp. 675682, 2001.

[21] J. Q. Han. Active Disturbance Rejection Control Technique - The Technique for Estimating and Compensating the Uncertainties, Beijing, China: National Defence Industry Press, 2009. (in Chinese)

[22] M. Y. Cui, D. H. Sun, Y. F. Li. Kinematic control algorithm for AGV with parameter uncertainties based on filtering backstepping. Control and Decision, vol. 28, no. 8, pp. 12001206, 2013.

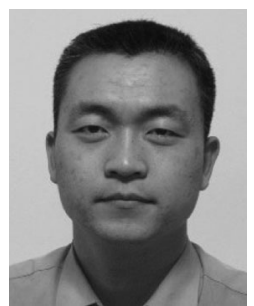

Fei Li received the B. Sc. and M. Sc. degrees from Engineering College, Air Force Engineering University, China in 2009 and 2011. He is a Ph. D. degree candidate in control science and engineering in Equipment Management and Safety Engineering College, Air Force Engineering University, China.

His research interests include sliding mode control and cyber-physical systems (especially the adaptive backstepping sliding mode control of flight dynamic system).

E-mail: lizhaodi726@163.com (Corresponding author)

ORCID iD: 0000-0001-8809-3704

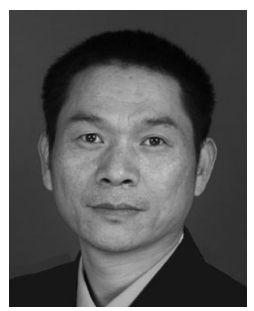

Jian-Bo $\mathbf{H u}$ received the B.Sc. and M. Sc. degrees from Engineering College, Air Force Engineering University, China in 1987 and 1990, and received the Ph. D. degree from Northwestern Polytechnical University, China in 1998. From 1998 to 2001 he did his postdoctoral research in Institute of Advanced Process Control, Zhejiang University, China. Now, he is a professor in Equipment Management and Safety Engineering College, Air Force Engineering University, China.

His research interests include robust adaptive control, variable structure control, and flight control system.

E-mail: hjbhjb6567@163.com

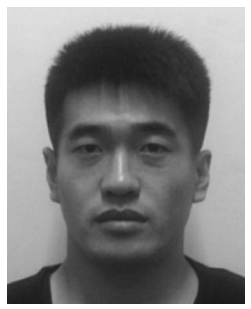

Lei Zheng received the B.Sc. and M.Sc degrees from Air Force Logistic College, China in 2010 and 2012. He is currently a Ph. D. degree candidate in control science and engineering in Equipment Management and Safety Engineering College, Air Force Engineering University, China.

His research interests include airworthiness management, variable structure control, and safety engineering.

E-mail: zhenglei-happy163.com

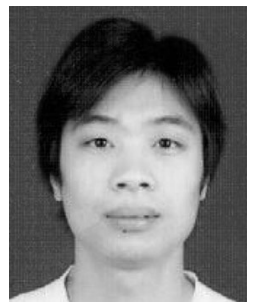

Jian-Hao Wang received the B.Sc. degree from $\mathrm{Xi}^{\prime}$ an Institute of Technology, China in 2006, the M. Sc. degree from Engineering College, Air Force Engineering University, China in 2009. He is a $\mathrm{Ph}$. D. degree candidate in control science and engineering in Aeronautics and Astronautics Engineering College, Air Force Engineering University, China.

His research interests include robust adaptive control, variable structure control, and intelligent system and control.

E-mail: hamilton-wang@sina.com 